\title{
Variability in knee radiographing: implication for definition of radiological progression in medial knee osteoarthritis
} Philippe Ravaud, Bruno Giraudeau, Guy-Robert Auleley, Jean-Luc Drape,
Benoit Rousselin, Laurence Paolozzi, Claude Chastang, Maxime Dougados

\begin{abstract}
Objectives-(1) To assess reproducibility of medial knee joint space width (JSW) measurement in healthy subjects and osteoarthritic (OA) patients. (2) To define minimal relevant radiological change in knee JSW based on the reproducibility of its measurement.

Patients and methods-(1) Healthy volunteers: in the first part of the study, 20 knees of healthy adult volunteers were radiographed in the weightbearing, anteroposterior extended view, twice, two weeks apart, using three different radiographic procedures: (a) without guidelines, (b) with guidelines and without fluoroscopy, (c) with guidelines and fluroroscopy. (2) Knee OA patients: in the second part of the study, 36 knees of OA patients were radiographed twice with guidelines and without fluoroscopy. JSW was measured blindly using a graduated magnifying glass. Based on the Bland and Altman graphic approach, cut off points defining minimal relevant radiological change are proposed.
\end{abstract}

Results-Standard deviation (SD) of differences in JSW measurement between two sets of knee radiographs in healthy subjects were $0.66 \mathrm{~mm}$ for radiography performed without guidelines, $0.37 \mathrm{~mm}$ for radiography performed with guidelines and without fluoroscopy, and 0.31 $\mathrm{mm}$ for radiography with guidelines and fluoroscopy. SD of differences in JSW measurement in $\mathrm{OA}$ patients were 0.32 mm for radiography performed with guidelines and without fluoroscopy. A minimal relevant change in JSW between two radiographs performed in healthy subjects can be defined by a change of at least 1.29 or $0.59 \mathrm{~mm}$ when radiographs are taken without guidelines, and with guidelines and fluoroscopy, respectively. When radiographs are taken with guidelines and without fluoroscopy, the change must be at least $0.73 \mathrm{~mm}$. A similar figure, $0.64 \mathrm{~mm}$ was observed in knee OA patients.

Conclusion-Definition of radiological progression varies greatly according to the radiographic procedure chosen. Use of guidelines reduces the threshold of progression required to consider that change between two measures is relevant.

(Ann Rheum Dis 1998;57:624-629)

At the present time, conventional radiographs are considered the most relevant outcome measure to assess the progression of osteoarthritis (OA) in clinical trials and epidemiological studies. ${ }^{1-3}$ Joint space width (JSW) measurement seems to be the most sensitive radiological criteria to detect changes over time, compared with other radiological variables such as bone response (for example, subchondral sclerosis, cysts, and osteophytes). JSW can be evaluated on different types of views: extended view, flexed view, semi-flexed view. Whatever the degree of flexion many sources of error or variability in JSW measurement have been identified. ${ }^{4}$ The influence of the measurement process (reader, measuring instrument) has been widely investigated. ${ }^{67}$ Several authors have also drawn attention to the influence of patient positioning and of the radiographic procedure in modifying the radiographic image and therefore JSW measurement. ${ }^{45}$ Most of the variability in JSW measurement can be attributed to variations in the radiographic procedure or joint positioning. ${ }^{4}$ Reproducibility of JSW measurement on multiple plain radiographs from the same person largely depends on the care taken in performing the radiography. The interest of precise guidelines has been emphasised to obtain comparable joint images on successive radiograhic visits. ${ }^{147}$ Some of these guidelines propose the use of fluoroscopy to facilitate capture of the joint space and determination of optimal foot rotation. ${ }^{4}$ However, in numerous centres fluoroscopy is not available and the real benefit obtained from using fluoroscopy remains to be evaluated.

When radiological abnormalities in $\mathrm{OA}$ are measured using a continuous variable such as JSW measurement (expressed in millimetres), we need to know whether an observed difference constitutes a relevant change. To be considered relevant - that is, related to a real 
structural change-changes in JSW measurement observed over time must exceed the changes induced by the variability inherent in repeating radiographs and in the measurement process.

The purpose of this study was (1) to assess the reproducibility of medial JSW measurement taken from conventional knee radiographs performed in healthy subjects and in OA patients using various procedures, (2) to propose a definition of minimal relevant radiological change, and to explore the influence of radiographing techniques on this minimal change.

\section{Methods}

RADIOGRAPHS

Healthy subjects

Twenty knees from 10 healthy male volunteers, mean age 35.4 (range 27-45) years and mean weight 73.3 (range 61-90) kg, were studied. Written informed consent was obtained from all participants. Radiographically, the knees of all volunteers were devoid of osteophytes or joint space narrowing. Radiographs were obtained with fast screen cassette film. Source to film distance was $115 \mathrm{~cm}$. Anteroposterior radiographs were taken with patients standing on both legs with their weight equally distributed.

Three imaging modalities were compared for each subject: (a) knee radiography performed without specific guidelines, (b) knee radiography performed using specific guidelines without fluoroscopy, (c) knee radiography performed using specific guidelines including the use of fluoroscopy.

Firstly, conventional weight bearing radiographs of both knees in full extension were taken. For these radiographs the usual radiographic procedure was used and no specific instructions were given to the technician.

Secondly, radiographs of each knee were taken separately using previously published guidelines ${ }^{4}$ but without the aid of fluoroscopy. According to these guidelines, the patient must be standing in full extension with the back of the knees as near as possible to the film plate. The distance between the back of the knees and the plate must be noted. The feet are rotated $15^{\circ}$ externally and the position of the feet is outlined on a sheet of paper. The central ray of the $x$ ray beam is centred on the joint space and inclined $5^{\circ}$ downward.

Thirdly, radiographs of each knee were performed separately following the same guidelines ${ }^{4}$ and with the aid of fluoroscopy. The patient must be standing in full extension with the back of knees as near as possible to the film plate. The distance between the back of knees and the plate is noted. With the aid of fluoroscopy, the lower limb is rotated so that the tibial spines appear centrally placed relative to the femoral notch. Thereafter, the position of the feet is outlined on a sheet of paper. The central ray of the $x$ ray beam is centred on the joint space and inclined downward to ensure that the medial tibial plateau is parallel to the $x$ ray beam. The angle of inclination is noted and reproduced on successive visits for ragiography.

Two weeks later, radiographs were repeated using the same radiographic procedures for the three imaging modalities. We assumed that over a two week period, no structural changes would have occurred.

For each healthy volunteer, 12 knee radiographs (two knees, three imaging modalities, at days 0 and 14) were taken, corresponding to a total radiation exposure equivalent to one skull radiograph or 20 days of natural background radiation. ${ }^{8}$ This estimation did not take into account radiation associated with fluoroscopy.

\section{$O A$ patients}

Twenty consecutive patients with symptomatic medial knee OA of the tibiofemoral medial compartment, mean age 61.4 (41-77) years and mean weight 73.9 (55-92) kg were recruited. The study was approved by the Cochin Institutional Review Board and written informed consent was obtained from each patient. Each patient was diagnosed as having radiographic OA of one or both knees if they were graded II or more according to the Kellgren and Lawrence grading system. Four patients had unilateral OA and 16 patients had bilateral OA. As a result, 36 knees were studied (15 grade II, 18 grade III, 3 grade IV).

Radiographs for each patient were performed using previously published guidelines but without the aid of fluoroscopy as described above. Two hours later, radiographs were repeated using the same radiographic procedure.

RADIOGRAPHIC ASSESSMENT

The subject's identification was masked with adhesive tape and replaced by a random code number on all radiographs. One reader (PR) assessed all radiographs blind to the radiographic modalities used, date of radiograph, and patient identification. Each knee was assessed separately. Using a one tenth millimetre graduated magnifying glass $(10 \times$ magnification), JSW was measured along a vertical line drawn from the midpoint of the medial femoral condyle to the medial tibial plateau. JSW was defined as the interbone distance between the distal convex margin of the femoral condyle and the floor of the tibial plateau. For healthy subjects, the reader assessed the 120 radiographs performed on day 0 and day 14 in one reading session and performed a second reading session one month later. For OA patients, the reader assessed the 72 radiographs (36 OA knees, two radiographs) performed during another reading session.

\section{STATISTICAL ANALYSIS}

The Bland and Altman graphic approach ${ }^{9} 10$ was used to assess the reproducibility of the radiographic procedures in healthy subjects and OA patients and the intra-reader reproducibility in measuring JSW in healthy subjects. This method focuses on the mean difference $\bar{D}$ between pairs of repeated measurements (obtained either from two visits for radiography 

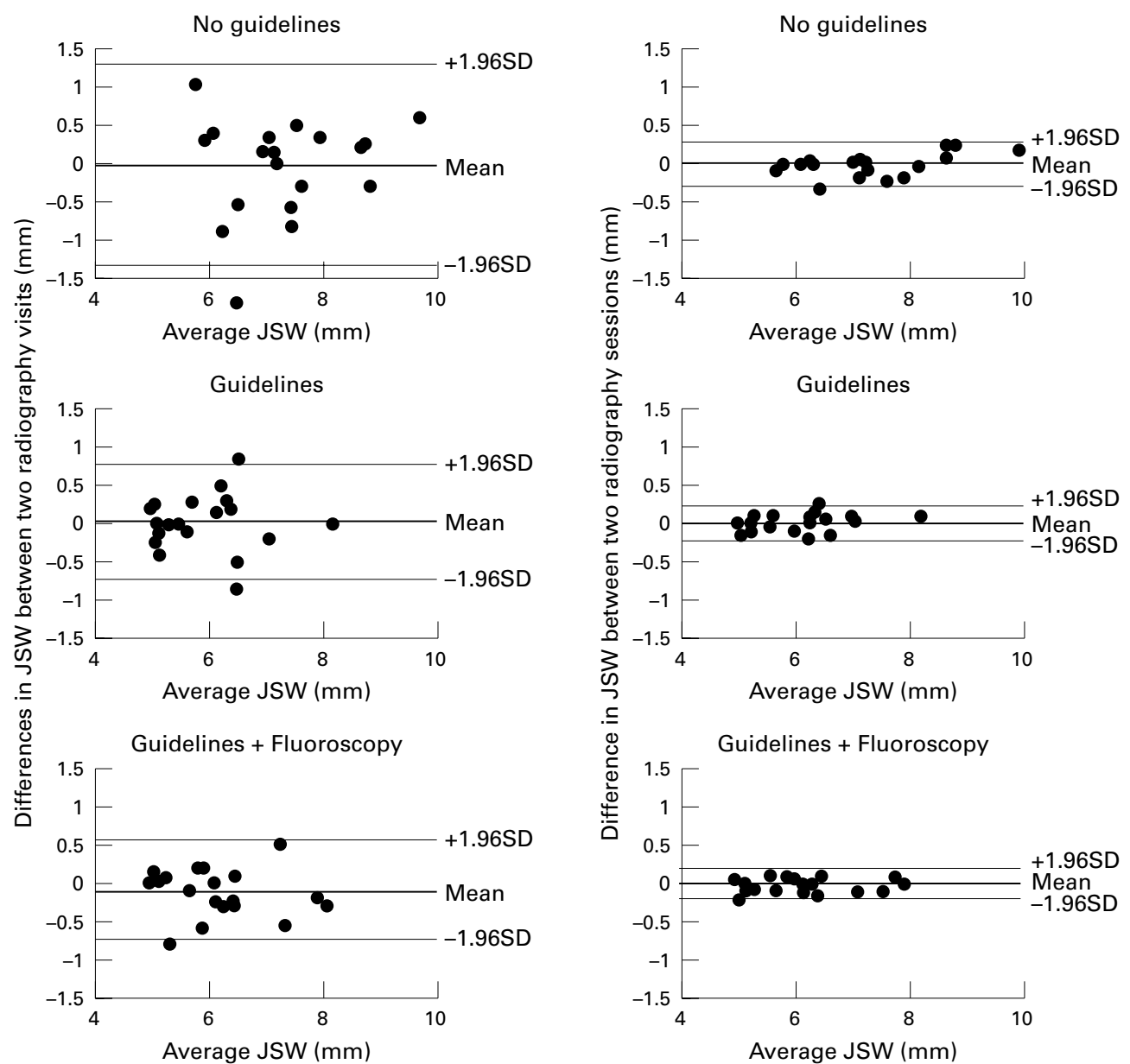

Figure 1 Healthy subjects. (Left) Differences in the measurement of $\mathcal{H S W}$ (in $\mathrm{mm}$ ) between radiographs performed on day 0 (first radiography visit) and 14 days later (second radiography visit) at the same reading session (first reading session) plotted against mean $\mathcal{Y S W}$ measurement for the three radiological procedures. (Right) Differences in the measurement of $7 S W$ (in $\mathrm{mm}$ ) for the same set of radiographs (first radiography visit) between first and second reading session plotted against mean FSW measurement. The horizontal lines in all panels indicate the mean difference (thick line) $\pm 1.96 S D$ (thin lines).

or from two reading sessions) and the standard deviation (SD) of these differences.

We propose to estimate JSW measurement variability by measuring JSW on serial radiographs performed over a short period. According to Bland and Altman, most disagreements in JSW measurement between two visits for radiography are expected to lie between limits called "limits of agreement". 910 These latter are defined as $\bar{D} \pm z_{(1-a / 2)} S D$ where $\bar{D}$ is the mean difference between pairs of repeated measurements obtained from two visits for radioraphy, and $z_{(1-\alpha / 2)}$ is the $(1-\alpha / 2)$ th percentile associated to normal distribution. In what follows, $\alpha$ will be fixed at 5,10 , and $20 \%$, thus leading to $z_{(1-a /}$ 2) values of $1.96,1.64$, and 1.28 respectively. This means that for a fixed risk, $\alpha$, we can consider that differences within $\bar{D} \pm z_{(1-\alpha / 2)} S D$ can be attributed to random errors (that is, errors induced by the variability inherent in repeating radiographs and in the measurement process) and therefore cannot be considered as organic changes. Conversely, differences greater, in absolute value, than $\bar{D} \pm z_{(1-\alpha / 2)} S D$ are considered as organic change. Assuming there is no organic change over a two week period, $\bar{D}$ is expected to be zero and we define $+z_{(1-\alpha / 2)} S D$ and $-z_{(1-\alpha / 2)} S D$ as cut off points. Such cut off points will allow in future studies to distinguish between patients who improved, worsened or remained stable over time. Indeed, a change over time in JSW greater than $+1.96 S D$ (if $\alpha$ is fixed at $5 \%$ ) probably reflects organic change rather than measurement error. Consequently, we will consider that a patient whose JSW increased by more than $1.96 S D$ is a patient who improved; a patient whose JSW reduced by more than $1.96 S D$ is a patient who worsened; a patient whose change in JSW is

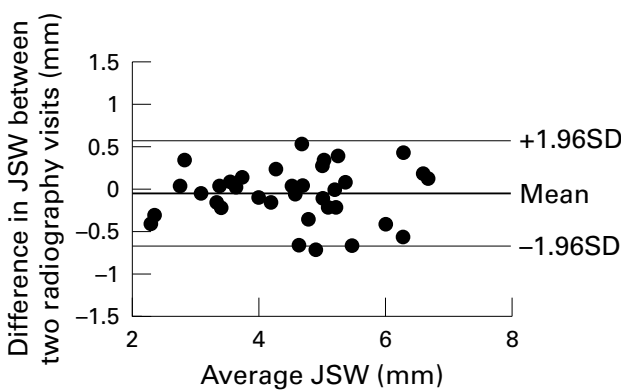

Figure 2 Knee OA patients. Differences in the measurement of $\mathcal{H S W}$ (in $\mathrm{mm}$ ) between radiography performed at hour 0 (first radiography visit) and two hours later (second radiography visit) plotted against mean joint space width measurement for radiography performed using guidelines but without fluoroscopy. The horizontal lines indicate the mean difference (thick line) $\pm 1.96 S D$ (thin line). 
Guidelines $v$ No guidelines

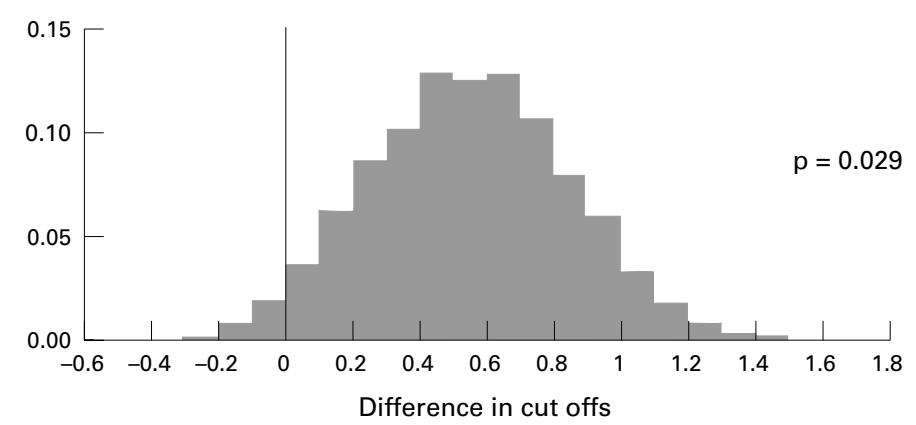

Guidelines + Fluoroscopy $v$ No guidelines

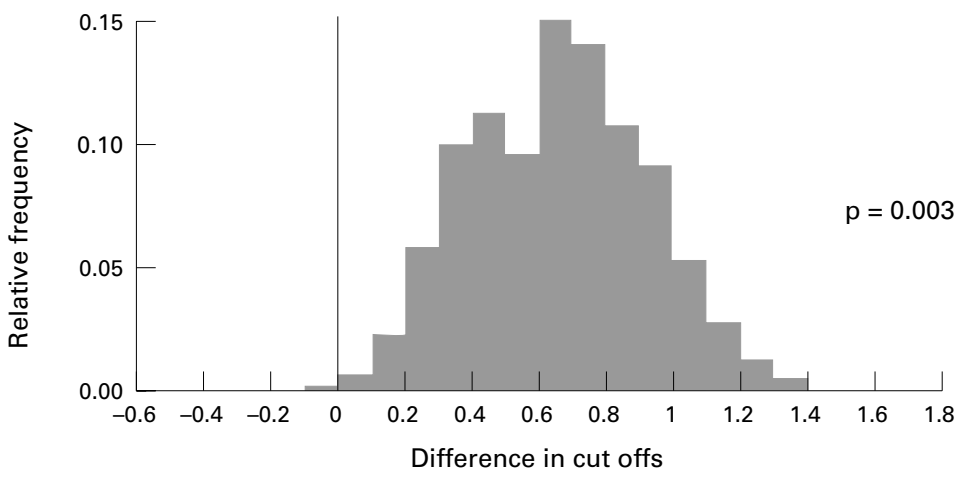

Guidelines + Fluoroscopy $v$ Guidelines

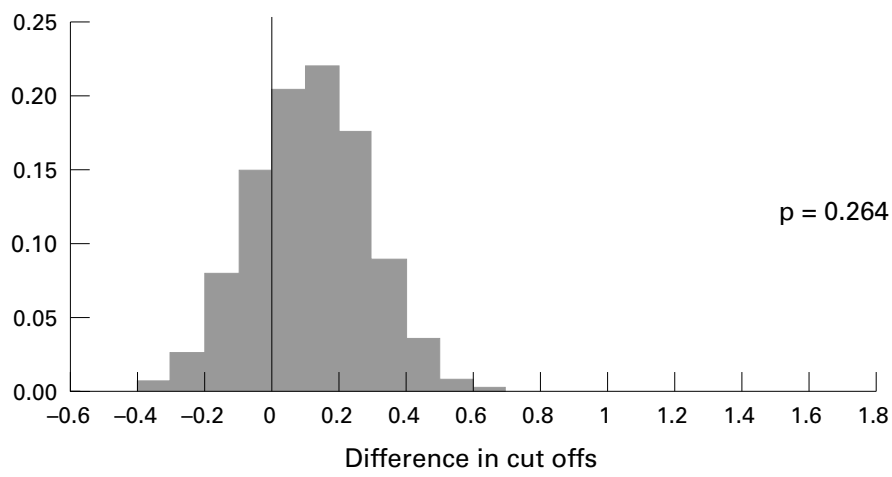

Figure 3 Histograms of bootstrap replications of the differences in cut offs associated to three radiological procedures. From each bootstrap sample, the three cut off radiographs performed without guidelines $\left(C O_{n}\right)$, with guidelines but without fluoroscopy $\left(C O_{C}\right)$, and with guidelines and fluoroscopy $\left(C O_{G \in \mathrm{F}}\right)$ are estimated as explained in the statistical analysis section. With a $5 \%$ risk, $C O_{G}$ is significantly smaller in absolute value than $C O_{n}$ $(p=0.029), C O_{G \Theta F}$ is significantly smaller than $C O_{n}(p=0.003)$, but $C O_{G \Theta F}$ is not significantly smaller than $C O_{G}(p=0.26)$.

between $-1.96 S D$ and $+1.96 S D$ is a patient who remained stable.

Cut off points associated to the three radiographic procedures named, respectively $\mathrm{CO}_{\mathrm{N}}$, $\mathrm{CO}_{\mathrm{G}}$, and $\mathrm{CO}_{\mathrm{G} \& \mathrm{~F}}$ for the radiographs performed without guidelines, with guidelines but without fluoroscopy, and with guidelines and fluoroscopy were then compared by pairs using bootstrap tests. ${ }^{11}$ Using a resampling scheme, the process was rerun 10000 times thus leading to 10000 estimates of $\mathrm{CO}_{\mathrm{N}}, \mathrm{CO}_{, \mathrm{G}}$ and $\mathrm{CO}_{\mathrm{G} \& \mathrm{~F}}$. This permits an empirical distribution to be made of the difference in two cut off points and then, considering the tail of the distribution, we derived the significant level of this difference.

To estimate the effect of guidelines and fluoroscopy on study power, sample size projections were made (see appendix). To test a loss in JSW over time, paired $t$ tests are appropriate. The variance we are interested in is then the variance of the difference in JSW, which can be viewed as the sum of the variance resulting from biological change over time (true variance) plus the variance because errors. True variance was fixed at 1 , which is a sensible value, ${ }^{12}$ and variance resulting from errors was estimated from the set of radiographs performed two weeks apart. We thus obtained ratios of sample size requirements associated to the different radiological procedures.

\section{Results}

REPRODUCIBILITY IN HEALTHY SUBJECTS

As shown in figure 1, SD of differences in the measurement of JSW between two sets of radiographs from the same people taken two weeks apart were $0.66 \mathrm{~mm}$ for radiographs performed without specific guidelines, 0.37 $\mathrm{mm}$ for radiographs performed using guidelines but without fluoroscopy and $0.30 \mathrm{~mm}$ for radiographs performed using guidelines including fluoroscopy.

In contrast, the SD of differences in the measurement of JSW between two readings of the same set of radiographs were $0.14 \mathrm{~mm}$ for radiographs performed without specific guidelines, $0.11 \mathrm{~mm}$ for radiographs performed using guidelines but without fluoroscopy, and $0.09 \mathrm{~mm}$ for radiographs using guidelines including fluoroscopy.

\section{REPRODUCIBILITY IN OA PATIENTS}

SD of differences in the measurement of JSW between two sets of radiographs from OA patients taken two hours apart were $0.32 \mathrm{~mm}$ for radiographs performed using guidelines but without fluoroscopy (fig 2).

DEFINITION OF PROGRESSION

In healthy subjects, with a 95\% level of confidence, the cut off points defining minimal relevant change in joint space width should be at least $1.29 \mathrm{~mm}$ for radiographs performed without guidelines, $0.73 \mathrm{~mm}$ for radiographs performed with guidelines but without fluoroscopy, and $0.59 \mathrm{~mm}$ for radiographs performed using guidelines and with fluoroscopy (table 1). Differences in cut off points were tested by pairs (fig 3). Using guidelines with or without fluoroscopy led to cut off points significantly smaller than the cut off point associated to radiographs performed without guidelines $(\mathrm{p}=0.003$ and $\mathrm{p}=0.029$ respectively). In contrast, with the use of guidelines, there was no significant difference with or without fluoroscopy $(p=0.26)$. Based on these results, it is now possible to evaluate the influence of these guidelines on the sample size of a study: the use of guidelines and fluoroscopy will spare only $5 \%$ of patients, compared with the use of guidelines alone; at variance the use of guidelines even without fluoroscopy will spare $32 \%$ of patients compared with the absence of use of guidelines. For example, if the sample size required to detect a change in JSW after one year is 100 subjects using guidelines and 
Table 1 Proposed cut off points for radiological progression according to the radiographic procedure and to the risk chosen (see results section for details). For example, in a study performed using guidelines without fluoroscopy, a change over 0.73 (at the 5\% risk level) will define structural progression of the disease

\begin{tabular}{|c|c|c|c|}
\hline & \multicolumn{3}{|c|}{ Cut off points for radiological progression $(\mathrm{mm})$} \\
\hline & risk $5 \%$ & risk $10 \%$ & risk $20 \%$ \\
\hline \multicolumn{4}{|l|}{ Healthy volunteers } \\
\hline No guidelines & 1.29 & 1.08 & 0.85 \\
\hline Guidelines without fluoroscopy & 0.73 & 0.61 & 0.47 \\
\hline Guidelines with fluoroscopy & 0.59 & 0.49 & 0.38 \\
\hline \multicolumn{4}{|l|}{ Knee OA patients } \\
\hline Guidelines without fluoroscopy & 0.62 & 0.52 & 0.41 \\
\hline
\end{tabular}

fluoroscopy, 105 subjects are required using guidelines without fluoroscopy and 132 without guidelines.

In OA patients, the cut off point defining minimal relevant change in JSW for radiographs performed using guidelines but without fluoroscopy should be at least $0.62 \mathrm{~mm}$ with a $95 \%$ level of confidence (table 1 ).

\section{Discussion}

This study provides data concerning the reproducibility of radiographing of the medial tibiofemoral compartment in healthy subjects and OA patients. Reproducibility of radiographing seems more influenced by standardisation of the radiographic procedure than by the use or not of fluoroscopy. This study also permits the proposal of a definition of minimal relevant radiological change. Cut off points differ greatly according to the care taken in re-radiographing. Cut off points obtained for radiographs performed without specific guidelines were approximately double those for radiographs performed following guidelines.

The results of the first part of the study were obtained in healthy volunteers who were radiographed twice at a two week interval. Some factors could limit the interpretation of this part of the study. Firstly, the number of knees radiographed is limited. Secondly, the subjects studied were not OA patients. However, the results observed in healthy volunteers are probably transposable to most OA patients except possibly to those with severe joint space narrowing (JSW less than $2 \mathrm{~mm}$ ). Moreover, for radiographs performed using guidelines but without fluoroscopy, variability appears similar for healthy subjects and OA patients with an SD of 0.37 and 0.32 respectively. Thirdly, the variability related to the radiographic procedure or joint positioning observed between serial radiographs performed two weeks apart is probably less than that observed between serial radiographs performed 12 or 24 months apart, particularly in OA patients. In OA patients, we can easily assume that modification of the activity of the disease (that is, presence of flare) induces difficulties to reproduce the recommended position. However, to decrease this variability, all data concerning radiographic procedures and joint positioning could be registered for each patient on a data form at the initial visit for radiography, and reproduced at each subsequent visit.

There is no consensus on the definition of relevant radiological progression in OA for a group of patients or an individual patient. The assessment of JSW using a continuous variable such as measurement of the interbone distance (evaluated in millimetres) at the narrowest point or at the midpoint is usually considered the primary end point to assess structural changes in OA. Estimates of minimal relevant radiological change permit results to be expressed as a dichotomous variable (number of patients who progress), and to calculate sample size requirements.

This cut off point can be defined either by consensual agreement or arbitrarily. Dieppe et al proposed that $2 \mathrm{~mm}$ is the minimal relevant radiological change for the evaluation of knee OA. ${ }^{13}$ It was also proposed that JSW measurement variability could be estimated by remeasuring the same radiographs, ${ }^{14}$ but this approach fails to include variations that occur between radiographs. Therefore, we propose a definition of this change based on analysis of reproducibility of radiographing. Radiological progression could be defined by a change in JSW greater than the limits of agreement in measurement of JSW between radiographs repeated over a short period (considering that no organic change can occur over this period). This procedure takes into account both variability in the radiographic procedure and variability in the measurement process.

The cut off points obtained varied greatly according to the imaging modalities and the risk level chosen. The cut off points can be greatly reduced with a standardised radiographic procedure. The use of fluoroscopy permits the reducion in the cut offs in healthy subjects but this reduction is not statistically significant. However, we cannot exclude that in OA patients, particularly in case of severe joint space narrowing, the influence of fluoroscopy should be greater than in healthy subjects. At a $5 \%$ risk level, radiological progression is defined as a change greater than $1.29 \mathrm{~mm}$ for radiographs performed without guidelines, and $0.73 \mathrm{~mm}$ and $0.59 \mathrm{~mm}$ respectively for radiographs performed using guidelines with or without the aid of fluoroscopy. These cut offs may vary according to the radiological procedure (radiology unit, care taken with repositioning, etc), the measuring instrument, the reader, and the sample of studied patients. Consequently, we propose in each study to repeat radiographs over a short interval (for example, one or two days apart) in a representative sample of patients (for example, 30 of 300) to determine the reproducibility of the radiographic procedure and to derive a specific cut off for the study in question. Our proposition is applicable to any study and requires only adding to the planned visit for radiography, one radiograph, performed and assessed in the study conditions, in one subgroup of studied patients.

Several criticisms could be applied to these cut offs. Firstly, the transformation of a continuous variable (that is, changes in $\mathrm{mm}$ per year) in a dichotomous variable (that is, progression yes/no) might lead to a decrease in the statistical power of a study. However, we consider that the expression of the results in the second way (progression yes/no) is much more 
clinically relevant and that the decrease in statistical power is counterbalanced by the gain in expression of the results. Secondly, the clinical relevance of such cut offs could be discussed. This approach did not permit the definition of a clinically relevant change, (that is, a change related to clinically relevant biological or structural outcome measure in OA). Our aim was focused on the definition of cut offs permitting the distinguishing of modifications potentially related to measurement errors from real or organic modifications of JSW.

In this study we chose as an example the extended view to illustrate our proposition of definition of minimal relevant radiological change. Several other views such as flexed and semi-flexed have been proposed to assess OA progression. The choice of the extended view was guided by the wide use of this view in clinical trials or epidemiological studies. However, such an approach should be applied to all types of view, in order to define (1) the respective influence of standardisation and fluoroscopy on their reproducibility, (2) a minimal radiological change using these views. It must be noted that the results observed in the medial compartment cannot be generalised to the whole knee and particularly to the lateral compartment.

To apply this method in daily practice, these results should be confirmed by other similar studies and the recommendations in performing radiography should be applied in all departments of radiology. Further longitudinal studies in OA patients are required to confirm the interest of minimal relevant radiological changes as defined in this study.

\section{Appendix}

In the paired $t$ test situation, the number of subjects in the group should satisfy ${ }^{15}$ :

$$
n=s^{2} \frac{\left(z_{1-\alpha}+z_{1-\beta}\right)^{2}}{\Delta^{2}}+\frac{1}{2} z_{1-\alpha}^{2}
$$

where $z_{1-\alpha}$ and $z_{1-\beta}$ are respectively the $\alpha$ th and $\beta$ th percentiles associated to a normal distribution

$\Delta$ is the difference which is expected to be detected from time 1 to time 2

$s^{2}$ is the variance of the differences.

This latter variance is the sum of the variance due to biological change (true variance) and the variance due to errors. Therefore, we can associate an estimate of this variance to each radiographic procedure:

$$
\begin{gathered}
s_{n}^{2}=s_{t v}^{2}+s_{e n}^{2} \\
s_{G}^{2}=s_{t v}^{2}+s_{e G}^{2} \\
s_{G F}^{2}=s_{t v}^{2}+s_{e G F}^{2}
\end{gathered}
$$

where the $n, G$ and $G F$ indices refer to the three radiographic procedures (no guidelines, guidelines without fluoroscopy, and guidelines with fluoroscopy, respectively), the $e$ index to the error terms and $s_{t v}^{2}$ to the true variance. To each of these variance is then associated a sample size: $n_{n}, n_{G}$ and $n_{G F}$. We thus obtain the following ratio:

$$
\begin{aligned}
& \frac{n_{n}-\frac{1}{2} z_{1-\alpha}^{2}}{n_{G F}-\frac{1}{2} z_{1-\alpha}^{2}}=\frac{s_{t v}^{2}+s_{e n}^{2}}{s_{t v}^{2}+s_{e G F}^{2}} \\
& \frac{n_{G}-\frac{1}{2} z_{1-\alpha}^{2}}{n_{G F}-\frac{1}{2} z_{1-\alpha}^{2}}=\frac{s_{t v}^{2}+s_{e G}^{2}}{s_{t v}^{2}+s_{e G F}^{2}}
\end{aligned}
$$

$s_{e n}, s_{e G}$ and $s_{e G F}$ are respectively estimated at 0.66 , 0.37 , and 0.31 . Assuming $s_{t v}^{2}$ equals 1 , which is a sensible value for a one year progression in JSW, and choosing $\alpha=5 \%$, we then deduce that when 100 subjects are needed using guidelines and fluoroscopy $\left(n_{G F}=100\right) 105$ are needed when using guidelines without fluoroscopy $\left(n_{G}=105\right)$ and 132 when no guidelines are used $\left(n_{n}=132\right)$.

Supported by grant from the Cassenne Laboratories, Puteaux, France.

1 Altman R, Brandt K, Hochberg M, Moskovitz R. Recommendations from a task force of the Osteoarthritis Research Society: Design and conduct of clinical trials in patients with osteoarthritis. Osteoarthritis Cartilage 1996;4:217with

2 Group for the Respect of Ethics and Excellence in Science (GREES): Osteoarthritis Section. Recommendations for the registration of drugs used in the treatment of osteoarthritis. Ann Rheum Dis 1996;55:552-7.

3 Dieppe P. Recommended methodology for assessing the progression of osteoarthritis of the hip and knee joints. Osteoarthritis Cartilage 1995;3:73-7.

4 Ravaud P, Auleley G R, Chastang C, Rousselin B, Paolozzi L, Amor B, et al. Knee joint space width measurement: An experimental study of the influence of radiographic procedure and joint positioning. Br J Rheumatol 1996;35:761-6.

5 Buckland-Wright J.C. Quantitative radiography of osteoarthritis. Ann Rheum Dis 1994;63:268-75.

6 Ravaud P, Chastang C, Auleley G R, Giraudeau B, Royant V, Amor B, et al. Assessment of joint space width in patients with osteoarthritis of the knee. A comparison of four measwith osteoarthritis of the knee. A comparison of four
uring instruments. J Rheumatol 1996;23:1749-55.

7 Buckland-Wright JC. Protocols for precise radio-anatomical positioning of the tibiofemoral and patello-femoral compartments of the knee. Osteoarthritis Cartilage 1995;3 (suppl A):71-80.

8 Royal College of radiologists. Making the best use of a department of radiology. Guidelines for doctors. London: RCR, 1993.

9 Altman DG, Bland JM. Measurement in medicine: the analysis of method comparison studies. Statistician 1983; 32:307-17.

10 Bland JM, Altman DG. Statistical methods for assessing agreement between two methods of clinical measurement. Lancet 1986;i:307-10.

11 Efron B, Tibshirani RJ. An introduction to the bootstrap. New York: Chapman and Hall, 1993.

12 Ravaud P, Giraudeau B, Auleley GR, Chastang C, Poiraudeau S, Ayral X, et al. Radiographic assessment of knee osteoarthritis: Reproducibility and sensitivity to change. J Rheumatol 1996;23:1756-64.

13 Dieppe PA, Cushnaghan J, Jasan MK, McCrae F, Watts I. A two-year placebo-controlled trial of non steroidal antiin-
flammatory therapy in osteoarthritis of the knee joint. Br J flammatory therapy in osteoar

14 Dougados M, Gueguen A, Nguyen M, Berdah L, Lequesne $\mathrm{M}$, Mazières $\mathrm{B}$, et al. Radiological progression of hip osteoarthritis: definition, risk factors and correlation with clinical status. Ann Rheum Dis 1996;55:356-62.

15 Machin D, Campbell MJ. Statistical tables for the design of clinical trials. London: Blackwell Scientific Publications, 1987. 